\title{
Polylactic acid/nano chitosan composite fibers and their morphological, physical characterization for the removal of cadmium(II) from water
}

\author{
Merin S. Thomas ${ }^{1,2,3}$ ～Prasanth K. S. Pillai ${ }^{1,4} \mid$ Marisa Faria $^{5}$ | \\ Nereida Cordeiro $^{5}$ | Lekshmi Kailas $^{6}$ | Nandakumar Kalarikkal ${ }^{1,7}$ | \\ Sabu Thomas ${ }^{1,8}$ | Laly A. Pothen ${ }^{1,3,9}$ (])
}

\author{
${ }^{1}$ International and Interuniversity Centre \\ for Nanoscience and Nanotechnology, \\ Mahatma Gandhi University, Kottayam, \\ India \\ ${ }^{2}$ Department of Chemistry, Mar Thoma \\ College, Tiruvalla, India \\ ${ }^{3}$ Department of Chemistry, C.M.S. \\ College, Kottayam, India \\ ${ }^{4}$ Food and Bioproduct Sciences, \\ University of Saskatchewan, Saskatoon, \\ Saskatchewan, Canada \\ ${ }^{5}$ Faculty of Exact Science and \\ Engineering, University of Madeira, \\ Funchal, Portugal \\ ${ }^{6}$ Materials and Surface Science Institute, \\ University of Limerick, Limerick, Ireland \\ ${ }^{7}$ School of Pure and Applied Physics, \\ Mahatma Gandhi University, Kottayam, \\ India \\ ${ }^{8}$ School of Chemical Sciences, Mahatma \\ Gandhi University, Kottayam, India \\ ${ }^{9}$ Department of Chemistry, Bishop Moore \\ College, Kallumala, India

\section{Correspondence} \\ Laly A. Pothen, Department of Chemistry, \\ C.M.S. College, Kottayam 686001, Kerala, \\ India. \\ Email: lapothan@gmail.com; lapothen@ \\ gmail.com
}

\begin{abstract}
This work discusses the fabrication of polylactic acid (PLA)/nano chitosan (nCHS) composite fibers by electrospinning method for $\mathrm{Cd}^{2+}$ metal ion adsorption from water. Here nCHS was synthesized by ionic gelation method and which is used as a reinforcement for PLA. The scanning electron microscopic analysis revealed that the addition $0.1 \mathrm{wt} \% \mathrm{nCHS}$ has decreased the fiber diameter as well as the secondary pore size and hence imparted unique properties to electrospun composite fibers. The positive zeta potential values for the composites indicated their higher stability, though; the inclusion of nCHS reduced the crystallinity of the neat membranes. The contact angle measurements showed that the hydrophilicity of the composite was increased up to $0.1 \mathrm{wt} \%$ nCHS, and hence the surface energy was increased. Inverse gas chromatography results suggested that the basic character of the composites has intensified with the increase in nCHS addition. The adsorption capacity of the neat electrospun PLA and PLA-nCHS composites for $\mathrm{Cd}^{2+}$ ions were investigated and studies revealed that adsorption capacity of the composite was two times faster (approximately 70\%) in comparison with neat PLA fibers. The increase in surface area as well as presence nCHS improved the adsorption capacity of the electrospun membrane.
\end{abstract}

\section{1 | INTRODUCTION}

Nano materials are finding renewed interest due to their novel properties, which are not seen in their conventional micro state counterparts. They are used in advanced applications in the fields of catalysis, medicine, electronics, optics, and membranes. ${ }^{[1-4]}$ Nowadays, membranes are highly utilized in waste treatment and water purification applications. ${ }^{[5]}$ The membranes can serve as a barrier in separating two distinct phases under pressure or concentration gradient, when in contact, and can transport one of the phases across the other. ${ }^{[6]}$ Electrospinning is one of the simplest and cheapest method to make nano-porous polymer membranes and offers a large surface area-to- 
volume ratio, high porosity, and small pore size in the electrospun non-woven mats, and could be employed for filtration, sensors, electrode materials, drug delivery, cosmetics, and tissue scaffolding. ${ }^{[7-10]}$

Traditionally, electrospun nanofibers are prepared from both natural and synthetic polymers, however, the growing concerns surrounding sustainability, biodegradability, and other environmental problems drives strong demand for natural polymers as an alternative to petroleum based feed stocks. ${ }^{[11]}$ Today, biodegradable polymers such as, polylactic acid (PLA), poly(glycolide), and poly(lactide-co-glycolide) etc., have drawn pronounced attention due to their high potential for biomedical applications, such as surgical sutures, drug carriers, tissue scaffolds, and implants for interior bone fixation. ${ }^{[12-14]}$ Polylactic acid is one of the most extensively researched, biodegradable thermoplastic polymers, which exhibit better mechanical strength than any other biopolymers, and degrades by de-esterification mechanism. ${ }^{[15,16]}$ It has been used to make membranes by electrospun method using a variety of solvents. ${ }^{[17]}$ However, the low stiffness of these electrospun fibers made it unwieldy to handle and may affect the processing techniques. Such type difficulties can be rectified by the incorporation of nano-reinforcements, and is a promising platform for advanced environmental applications, such as liquid filtration and particulate separation for water treatment and environmental remediation, respectively. ${ }^{[18]}$ The addition of distinct types of nano scale materials such as nano clay, carbon nano tubes, chitosan etc., with electrospun PLA fibers can tailor their properties for specific end uses such as packaging, biomedicals, and purification etc. Among them, chitosan is widely accepted for advanced biomedical and environmental applications (reference) due to its non-toxicity and barrier properties. ${ }^{[19,20]}$ Chitosan, the linear co-polymer of $\mathrm{N}$-acetylglucosamine (GlcNAc) and glucosamine (GlcN) residues, is linked via $\beta-1$, 4-glycosidic bonds, and is obtained via the deacetylation of chitin. Chitosan has countless potential for sorption of metal ions due to the presence of amino and hydroxyl groups in its chemical structure.

Present study explored the fabrication of porous electrospun mats of polylactic acid incorporated with chitosan nanoparticles, synthesized by ionic gelation method for cadmium(II) removal. For studying the adsorption capacity of the membrane, we analyzed the surface morphology, crystallinity, and thermal properties of the electrospun membranes. It is hypothesized that the introduction of nano chitosan (nCHS) would decrease the fiber diameter as well as the pore size of the membrane system. To this date no literature is available on the use of nCHS as filler in the electrospinning process of PLA.

\section{MATERIALS AND METHODS}

\section{1 | Materials}

PLA used in this study was supplied by Shenzhen Bright China Industrial Co. Ltd, Guangdong, China and has a density of $1.24 \mathrm{~g} / \mathrm{cm}^{3}$ reported by the supplier and the chitosan (medium molecular weight) was purchased from Marine chemicals, Cochin, India. The solvents used, chloroform $\left(\mathrm{CHCl}_{3}\right)$ (99\% purity), acetic acid (>99\% purity) were purchased from Merck Chemicals, Mumbai (India). Sodium tripolyphosphate (TPP) used for the synthesis of chitosan nanoparticles purchased from SigmaAldrich, Bangalore (India). Double distilled water was used for all synthesis. Methane ( $>99.99 \%$ purity) supplied by Air Liquide Company, Paris, France. Acetonitrile, ethyl acetate, ethanol, acetone, and tetrahydrofuran all gas chromatography (GC) grade (>99\% purity) were purchased from Sigma-Aldrich.

\section{2 | Synthesis of chitosan nanoparticle (nCHS)}

Chitosan nanoparticles were prepared by the ionic gelation method. ${ }^{[21]}$ Five milligrams of chitosan (CHS) was weighed in a clean, dry conical flask. Then, $100 \mathrm{~mL} 1 \%$ acetic acid solution was added and the solution stirred for one and a half hour using a magnetic stirrer. The solution was filtered and $100 \mathrm{~mL}$ TPP (sodium tripolyphosphate) was added to the solution, drop by drop, using a burette and stirred for an hour. This solution contains micro as well as nanoparticles, from which, the nanoparticles were separated using ultracentrifugation at $4000 \mathrm{rpm}$, and at $4^{\circ} \mathrm{C}$. Further the supernatant was dialyzed for 24 hours to maintain neutral $\mathrm{pH}$. The dialyzed samples were freeze dried (Daihan Labtech, Korea) to obtain the nCHS particles.

\section{3 | Electrospinning}

The Electrospinning method (Holmarc, Kochi, India) was used to prepare neat PLA and PLA/nCHS fibers. Separately $12.5 \mathrm{wt} \%$ PLA and 0.05, 0.1, 0.5, $1 \mathrm{wt} \% \mathrm{nCHS}$ in chloroform was prepared and the two solutions were mixed well using a magnetic stirrer $(1,000 \mathrm{rpm})$ for 24 hours and then ultrasonicated (MSW-269) for 20 minutes just before electrospinning. The distance between the needle tip and metallic collector was adjusted to $15 \mathrm{~cm}$ and kept at an applied voltage of $15 \mathrm{kV}$ over the gap. The PLA-nCHS solution was fed by syringe pump (with a flow rate of $1 \mathrm{~mL} /$ hour). 


\subsection{Fourier transform infrared spectroscopy}

Fourier transform infrared (FTIR) spectra of the membranes were obtained using SPECTRUM 400 spectrometer equipped with a PIKE Gladi attenuated total reflectance system and deuterated triglycine sulphate (DTGS) system on a diamond crystal with 15 scans at $4 \mathrm{~cm}^{-1}$ resolution. Each spectrum was obtained within the range of $4,000-500 \mathrm{~cm}^{-1}$.

\section{5 | Microscopy}

The surface morphology of the samples was analyzed by scanning electron microscopy (SEM) imaging. The micrographs of the spun fibers were taken in JEOL, Model JSM 6390 (Japan) electron microscope with an accelerating voltage of $10 \mathrm{kV}$. The samples were coated with platinum to avoid the electrostatic charge dissipation. Image $\mathrm{J}$ (version 1.44) software was used for the characterization of the SEM micrographs.

Field emission scanning electron microscopy (FESEM) images were obtained using a HITACHI SU 6600 Microscope with an accelerating voltage of $20 \mathrm{kV}$. The surfaces of the PLA, PLA-nCHS nanocomposite membranes were studied using FESEM.

Transmission electron microscopy (TEM) was performed on a JEOL JEM-2010 high resolution transmission electron microscope at an acceleration voltage of $120 \mathrm{kV}$.

\section{6 | X-ray diffraction (XRD) analysis}

The crystallinity of the materials (electrospun membranes after the incorporation of chitosan and nanochitosan) was examined using a wide-angle X-Ray diffractometer (D8- Advance, Bruker AXS, Germany). The measurements were done at $2 \theta=10^{\circ}$ to $50^{\circ}$, with Ni filtered $\mathrm{CuK} \alpha$ radiation source, having wavelength $1.54 \AA$ operated at $40 \mathrm{kV}$ voltage and $25 \mathrm{~mA}$ current.

\section{$2.7 \quad$ Tensile measurements}

Tensile testing of the electrospun mats were carried out according to ASTM D 882 using Tinus Olsen H50 KT Universal Testing Machine by applying a 500-N load cell at a crosshead speed of $1 \mathrm{~mm} / \mathrm{min}$. All the samples were cut into rectangles of dimensions of $6 \times 1 \mathrm{~cm}^{2}$ and vertically mounted in between two mechanical gripping units of the tester, leaving a 3-cm gauge length for mechanical loading. The sample thicknesses were measured with an electronic screw gauge having a precision of $1 \mu \mathrm{m}$. The average values of tensile property were obtained from the results of five tests and expressed as the mean \pm SD.

\section{8 | Zeta potential measurement}

Zeta potential measurement was done using Zeta potential Analyzer (Zetasizer 300 HAS; Malvern Instruments, Worcestershire, UK). All measurements in this study were taken at a temperature of $25^{\circ} \mathrm{C}$. A minimum of three replicate measurements on each sample were taken to check for result repeatability.

\section{9 | Thermal analysis}

The TGA measurements were carried out on a Q500 model (TA Instruments, New Castle, DE). Approximately 8.0-15.0 mg of sample was loaded into the open TGA platinum pan then heated from $25^{\circ} \mathrm{C}$ to $600^{\circ} \mathrm{C}$ at a constant rate of $10^{\circ} \mathrm{C} / \mathrm{min}$. The thermal degradation data was analyzed using TA Universal analysis software (version 3).

The thermal transition behavior of the samples was investigated using a Q200 model (TA Instruments) by modulated differential scanning calorimetry (DSC) following ASTM E1356-03 standard. The sample (3.0-6.0 mg) in hermetically sealed aluminum DSC pan was first equilibrated at $25^{\circ} \mathrm{C}$ and heated to $150^{\circ} \mathrm{C}$ at $10^{\circ} \mathrm{C} / \mathrm{min}$ (first heating cycle). The sample was held at that temperature for 10 minutes and then cooled down to $0^{\circ} \mathrm{C}$ at $10^{\circ} \mathrm{C} / \mathrm{min}$, and subsequently reheated to $150^{\circ} \mathrm{C}$ at the same rate (second heating cycle). Modulation amplitude and period were $\pm 1^{\circ} \mathrm{C}$ and 60 seconds, respectively. The "TA Universal Analysis" software was used to analyze the TGA and DSC thermograms. The characteristics of non-resolved peaks were obtained using the first and second derivatives of the differential thermogravimetry (DTG) and differential heat flow.

\subsection{0 | Contact angle measurement}

The surface contact angles were measured by SEO Phoenix 300 (version 8). The electrospun membranes were positioned on the stage of the instrument. The contact angle is measured as the tangent angle formed between a liquid drop and its supporting surface. All the experiments were performed at room temperature. The contact angles could be calculated by the software through analyzing the shape of the drop. The contact angle $\theta$ was an average of 50 measurements. Contact angle measurements of nanocomposites of PLA with chitosan were done at room temperature with distilled water. Total surface 
free energy from these measurements was calculated. The contact angles were measured for each sample for at least six to ten times.

\subsection{1 | Inverse gas chromatography}

Inverse gas chromatography (IGC) measurements of the nanocomposites fibers of PLA/nCHS were carried out on a commercial inverse gas chromatography equipped with flame ionization and thermal conductivity detectors. The IGC system was fully automatic with SMS IGC Controller V 1.8 Control Software. Data analysis was done using IGC Standard Analysis Suite V 1.3 and IGC Standard Analysis Suite V 1.21. The columns used were standard glass silanized (dimethyldichlorosilane; Repelcote BDH, Poole, UK) with $300 \mathrm{~mm}$ length and an internal diameter of $2 \mathrm{~mm}$. The samples were packed in the columns by vertical tapping for 2 hours using the SMS sample packing device and conditioned over-night at $40^{\circ} \mathrm{C}$ followed by 2 hours at temperature analysis. The dispersive component of the surface free energy was determined by applying four $n$-alkanes: heptane, octane, nonane, and decane at $25^{\circ} \mathrm{C}$. Acetonitrile, ethyl acetate, ethanol, and tetrahydrofuran were the polar molecules used for the determination of the specific free energy and acid-base surface character. The $n$-octane was used as the probe molecule to determine the parameters of isotherm, permeability, and diffusion experiments. All the measurements were carried out at $0 \%$ relative humidity, a helium flow rate of $10 \mathrm{~mL} / \mathrm{min}$, and in duplicate, producing an experimental error below $4 \%$. Methane was used as reference molecule for the calculation of dead time and helium was used as carrier gas with a flow rate of $10 \mathrm{~mL} / \mathrm{min}$.

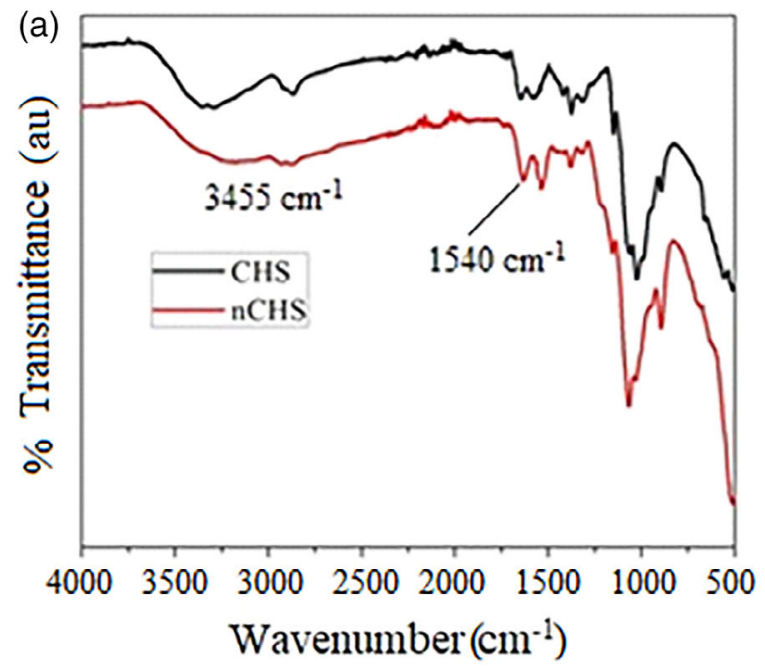

\subsection{2 | Determination of heavy metal ion concentration}

The solutions containing desired concentration of $\mathrm{Cd}^{2+}$ was prepared by directly dissolving cadmium chloride $\left(\mathrm{CdCl}_{2} \cdot 5 \mathrm{H}_{2} \mathrm{O}\right)$ in deionized water. The adsorption experiments were carried out by suspending $0.1 \mathrm{~g}$ of PLA or PLA-nCHS membranes in $50 \mathrm{~mL}$ cadmium chloride solutions at room temperature under continuous stirring. The amount of the adsorbed and unadsorbed $\mathrm{Cd}^{2+}$ in the solution was determined by measuring the concentration of the metal ions by Atomic Absorption Spectrophotometer (Perkin Elmer model AA 400), at every 30 minutes interval. Concentration of the metal ion (\%Metal) removed by the electrospun membranes were determined by Equation (1). Where $R$ is the percentage of $\mathrm{Cd}(\mathrm{II})$ adsorbed by membrane, $C_{\mathrm{T}}$ is the total concentration of metal ions in $\mathrm{mg} / \mathrm{L}$ and $C_{\mathrm{e}}$ is the concentration of metal ions at time $\mathrm{t}$ in $\mathrm{mg} / \mathrm{L}$.

$$
R(\%)=\left(C_{\mathrm{T}}-C_{\mathrm{e}}\right) / C_{\mathrm{T}} * 100
$$

\section{3 | RESULTS AND DISCUSSION}

\section{1 | FTIR characterization}

Chitosan nanoparticles (nCHS) were prepared by the ion gelation method from CHS solutions. The cationic and anionic character of CHS and TPP, respectively, make them suitable to interact through ionic exchange and, consequently, obtain the cross-linked nanoparticles. Figure 1a shows the FTIR spectra of CHS and nCHS. The peaks at $3455 \mathrm{~cm}^{-1}$ in CHS and nCHS are due to the

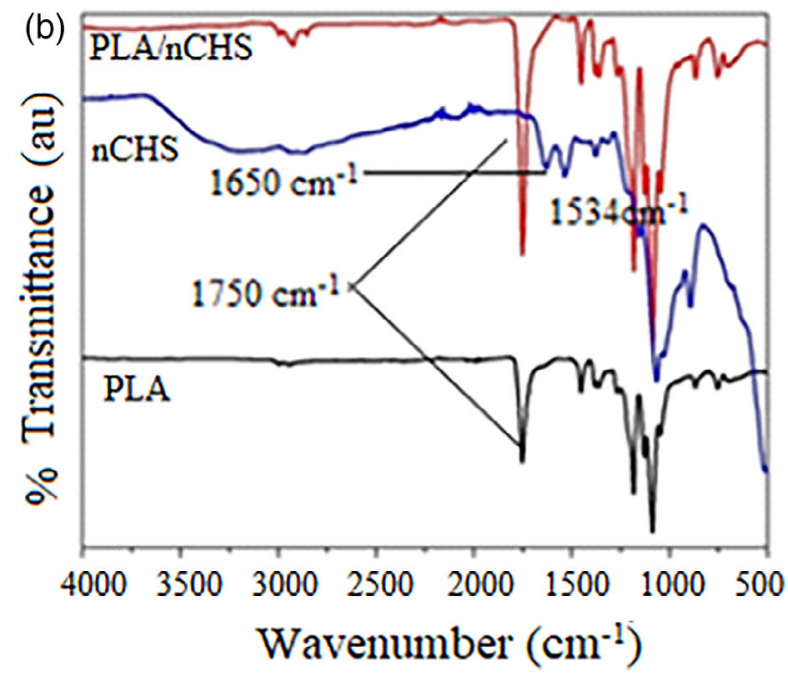

F I G URE 1 The fourier transform infrared (FTIR) spectra of (a) chitosan (CHS) and nano chitosan (nCHS) and (b) polylactic acid (PLA), PLA-nCHS composite, and nCHS [Color figure can be viewed at wileyonlinelibrary.com] 
$-\mathrm{NH}$ and $-\mathrm{OH}$ stretching vibrations. Peak at $1540 \mathrm{~cm}^{-1}$ is assigned to $\mathrm{N}-\mathrm{H}$ bending vibration of amide group. The characteristic peaks for saccharide structure of chitosan appeared in between 1,000 and $1,250 \mathrm{~cm}^{-1}$. From the spectra of CHS and nCHS (Figure 1a), it was confirmed that no structural changes occurred after the formation of nCHS. ${ }^{[22]}$

Figure $1 \mathrm{~b}$ represents the FTIR spectra of the PLA, nCHS, and PLA-nCHS composites. The ester group in PLA was confirmed by the characteristic band at $1750 \mathrm{~cm}^{-1}$. Note that the characteristic ester peak was present in the neat fiber as well as in composite fibers. Two characteristic peaks appearing at $1650 \mathrm{~cm}^{-1}$ (C ONHR) and $1,534 \mathrm{~cm}^{-1}\left(\mathrm{NH}_{2}\right)$ corresponds to the $\mathrm{N}-\mathrm{H}$ bend vibrations in primary amines, while, the amide $\left(\mathrm{C}-\mathrm{N}\right.$ ) band appears around $1,331 \mathrm{~cm}^{-1}$ (Zhao et al. 2014). The stretching vibrations $-\mathrm{OH}$ and $-\mathrm{NH}$ of chitosan were found at $3100-3500 \mathrm{~cm}^{-1}$. The prepared PLA-nCHS composites is characterized by the absence of
$-\mathrm{OH}$ and $-\mathrm{NH}$ stretching vibrations of nCHS at $3100-3500 \mathrm{~cm}^{-1}$, which is the typical feature of chitosan. This is because of the very low concentration of nCHS in composites. ${ }^{[23,24]}$

\section{2 | Morphological characterization of composites by microscopy}

The PLA/nCHS composites were evaluated by SEM in order to acquire information about their morphology which is also an important parameter for their possible/ specific applications. SEM analysis was used to assess the fiber diameters and to reveal porous nature in the nanofibrous structure. Figure 2 exhibits the SEM micrographs of the surface of electrospun neat PLA and PLA/nCHS at different concentration $(0.05,0.1,0.5$, and $1 \mathrm{wt} \%)$. The PLA/nCHS composites had a markedly smaller average diameter compared to homogenous PLA,
F I G URE 2 Scanning electron microscopy (SEM) images of (a) neat polylactic acid (PLA) fibers,

(b) $0.05 \mathrm{wt} \%$ nano chitosan (nCHS) loaded, (c) $0.1 \mathrm{wt} \%$ nCHS loaded,

(d) $0.5 \mathrm{wt} \%$ nCHS loaded, and

(e) $1 \mathrm{wt} \%$ nCHS loaded PLA fibers, respectively
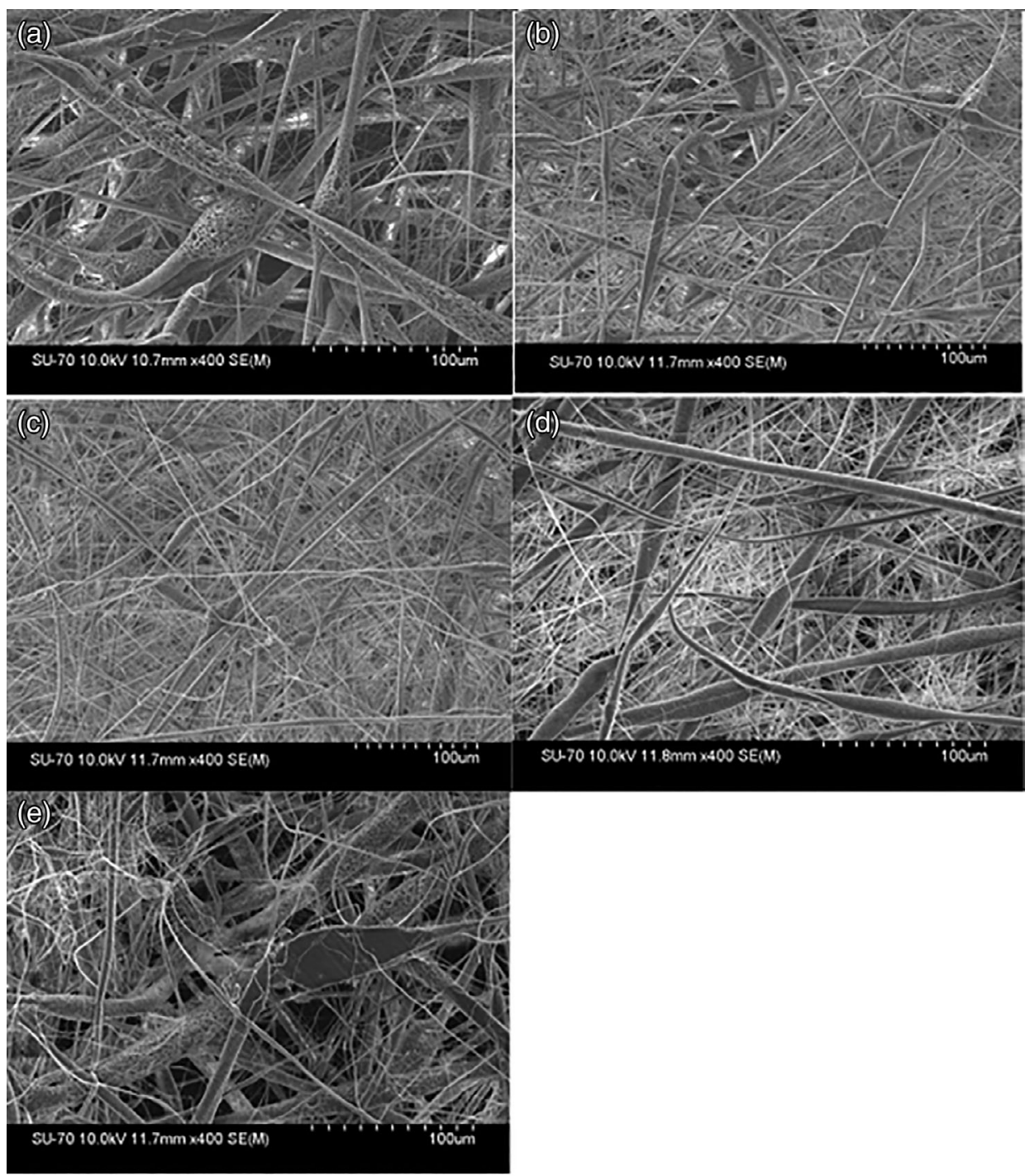
which occurred due to the solution viscosity increment by the controlled addition of nCHS into PLA. As seen in Figure 2, at lower filler (nCHS) concentration, a reduction in filler diameter was observed with PLA/nCHS composites. However, at higher filler additions, due to the agglomeration of nCHS particles as well as the higher viscoelastic forces of the solution, which resisted the axial stretching during whipping, resulted in larger fiber diameter. ${ }^{[25]}$ That means increase in nCHS content will lead to increase in viscosity while after $0.1 \mathrm{wt} \%$ concentration the viscosity become very high, as a result the polymer solution face a difficulty to stretch and resulting in larger fiber diameter. Figure 3 represents the histogram of average fiber diameter of neat PLA and composites with 0.1 nCHS loading. It reveals that the fiber diameter decreases drastically from 2000 to $1,000 \mathrm{~nm}$ range.

Figure $4 \mathrm{a}, \mathrm{b}$ represents the FESEM image of neat PLA and PLA loaded with $0.1 \mathrm{wt} \%$ CHS, respectively. The characteristic secondary porous structure of PLA was visible in both the images (see Figure 4a, b). The secondary porous structure can be believed to have been developed during the electrospinning process. The evaporative cooling of solvent on the surface of the fibers and the condensation of water vapor present in the atmosphere on the fiber surface, during the path of the polymer jet from needle tip to collector, creates an imprint on the fiber surface called breath figures (see Figure 4). The breath figures indicates the evaporative cooling of solvent on the surface of the spun fibers, which might have happened during the path of the polymer jet from needle tip to collector, where the water vapor present in the atmosphere condenses on the surface of the fibers creating an imprint on the surface. ${ }^{[24]}$ Such type phenomena was previously observed with electrospun PLA systems, and indicated that the surface morphology of the systems are highly impacted by the solution chemistry of polymer solvent system and their phase separation dynamics. ${ }^{[2,26]}$ Another reason for the formation of such type secondary structure might be
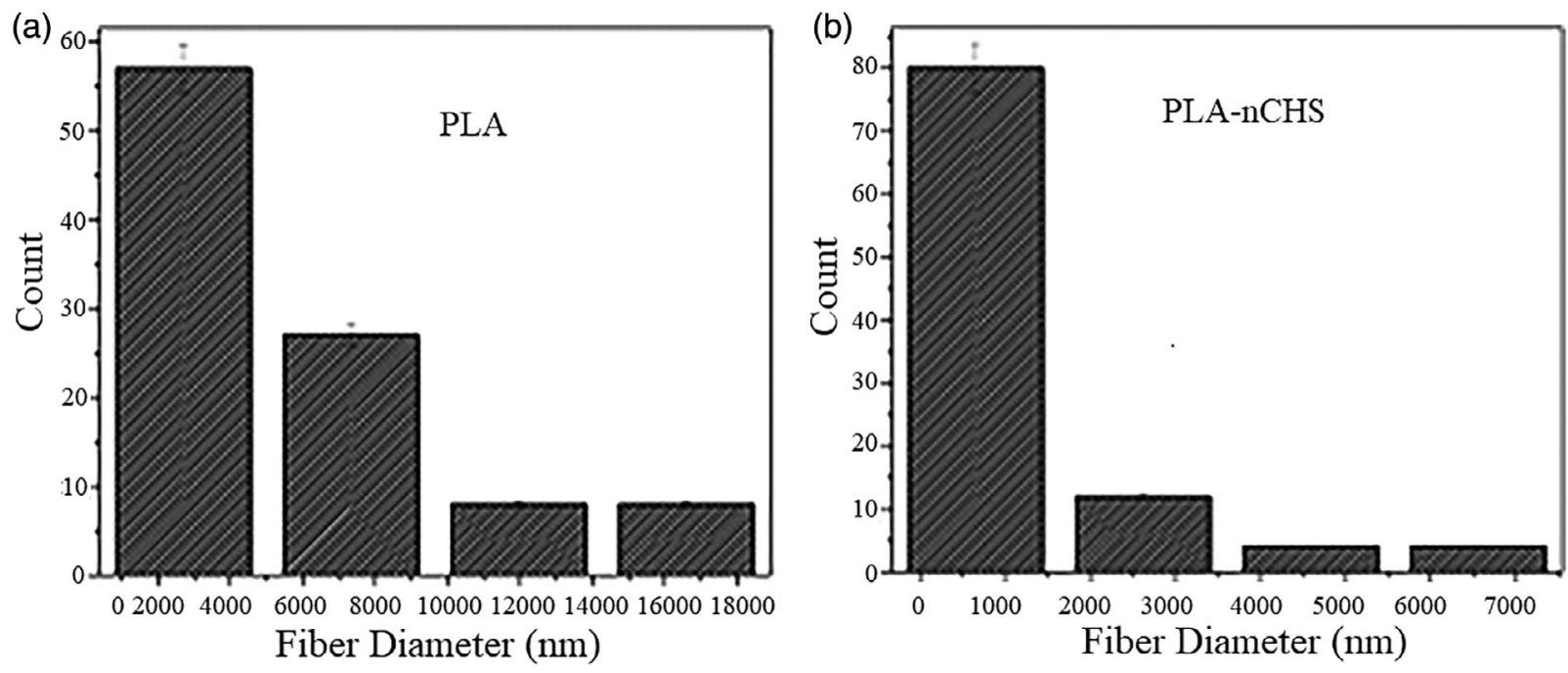

F I G U RE 3 Fiber diameter distribution histograms of (a) polylactic acid (PLA), (b) PLA + 0.1 wt\% nano chitosan (nCHS)

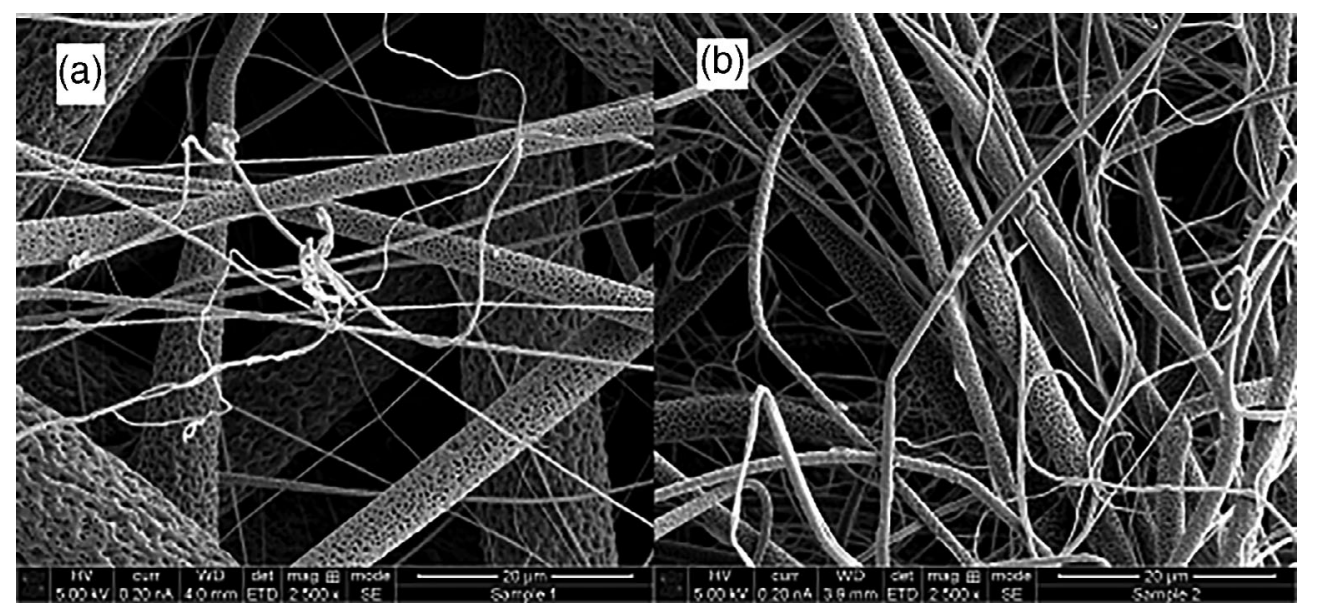

FI G URE 4 Field emission scanning electron microscopy (FESEM) images of (a) neat polylactic acid (PLA) and (b) PLA loaded with $0.1 \mathrm{wt} \%$ chitosan (CHS) 
(a)

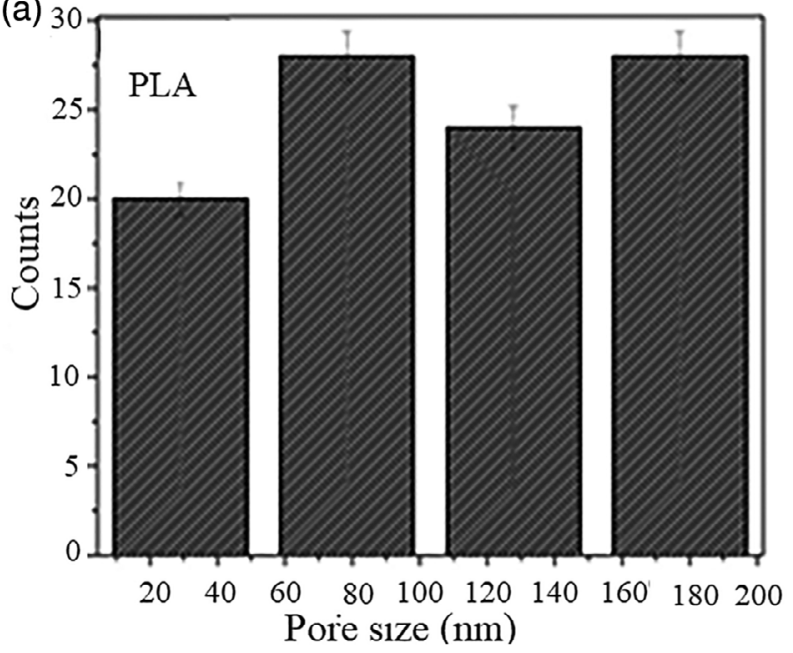

(b)

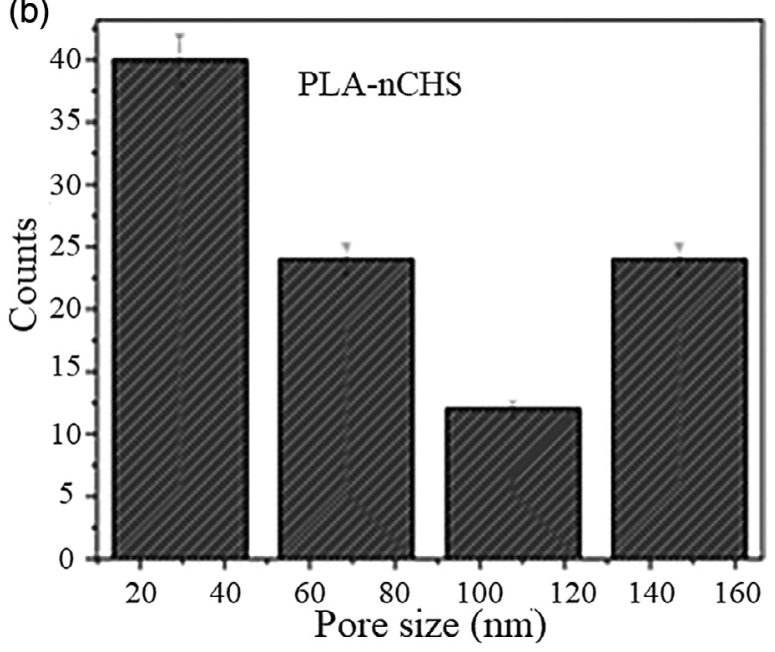

F I G U R E 5 Histograms representing the pore size of FESEM images of (a) neat polylactic acid (PLA) and (b) PLA loaded with 0.1 wt\% nano chitosan (nCHS)

(a)

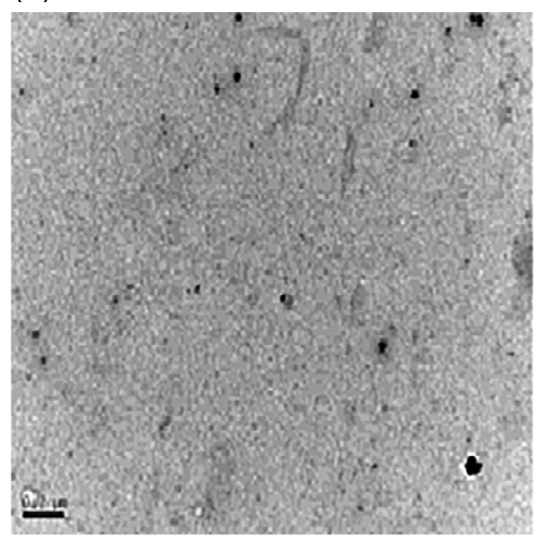

(b)

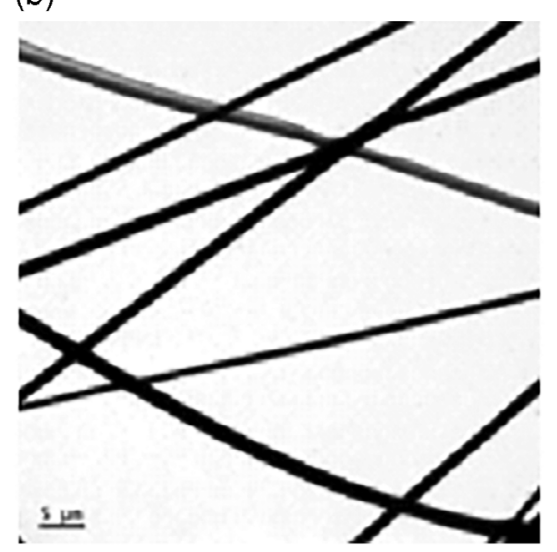

(c)

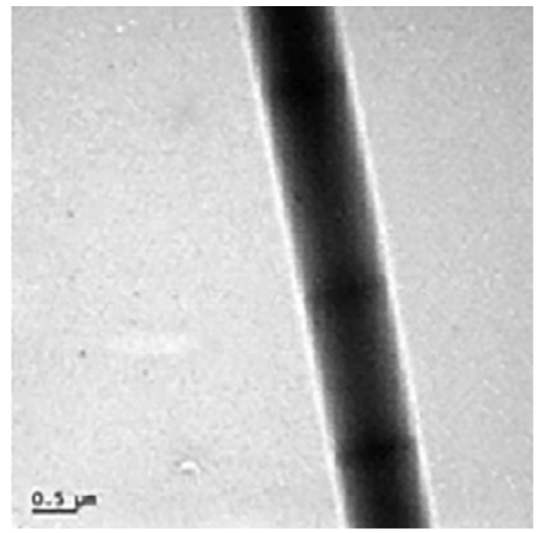

F I G U R E 6 Transmission electron microscopy (TEM) images of (a) nano chitosan (nCHS), (b) polylactic acid (PLA), and (c) PLA-nCHS composite

the spinodal decomposition resulting in phase separation. ${ }^{[27]}$ That means during electrospinning process, there generates polymer-rich and solvent-rich regions due to the thermodynamic instability of the spinneret. The polymer rich region hardens rapidly than the solvent rich region and hence pores are formed. Both these effects contribute to the porosity of the individual fibers. ${ }^{[24]}$ It was reported before that the interconnected porous structure of the electrospun fibers was generally attributed to phase separation by the spinodal decomposition mechanism. ${ }^{[28]}$

The pore size of the neat fiber and composites were measured using image J software, version 1.44, and it was found that there is a drastic decrease in the diameter of the pore in composites. Figure 5 shows the histogram of average pore size of neat PLA with $0.1 \mathrm{wt} \%$
nCHS loading, which revealed that the pore size dramatically decreased to the nanoscale range in the case of composites. TEM images were used for getting information regarding alignment of nCHS particles on the fiber surface. From the TEM images (Figure 6), the average sizes of the spherical nCHS particles were found to be $21.9 \mathrm{~nm}$ (Figure 6a). The TEM image of PLA-nCHS composite (Figure 6c) indicated that the chitosan particles were fully encapsulated into the PLA matrix.

\section{3 | X-ray diffraction analysis}

The effect of nCHS on the crystalline behavior of electrospun polylactic acid membranes was studied by 
XRD analysis. The XRD patterns of the neat PLA membrane and nCHS/PLA nanocomposites are shown in Figure 7. The unprocessed PLA possesses a semi crystalline nature with two characteristic peaks at $16.8^{\circ}$ and $19.2^{\circ} \cdot{ }^{[29]}$ But the XRD plots of electrospun PLA and the PLA-nCHS membranes represent amorphous character, which strongly suggest that the molecular organization was intensely influenced by electrospinning process. ${ }^{[30]}$ This is due to the stretching and rapid solidification of the polymer solution, which almost completely hinders the crystallization behavior of PLA. ${ }^{[31]}$ However, the diffractogram of nCHS does not have these peaks. This is an indication of the destruction of crystalline structure of chitosan during ionic gelation. ${ }^{[32]}$ On comparison with the neat PLA, electrospun membrane, there is a reduction in crystallinity and the increase in amorphous nature with increase in filler content, which might be due to the complexation between polymer and filler.

\section{4 | Tensile strength}

The stress-strain curves for electrospun PLA/ nCHS nanocomposites are presented in Figure 8 and the mechanical properties are presented in Table 1 . The tensile strength of the PLA/nCHS membranes increased with increasing nCHS content upto $0.1 \mathrm{wt} \%$, while further increasing the nCHS content has decreased the tensile strength of the membranes (Figure 8). At lower concentration of nCHS the nano composite showed superior tensile strength compared with the neat PLA membrane. At higher loading, after $0.1 \mathrm{wt} \%$ the tensile

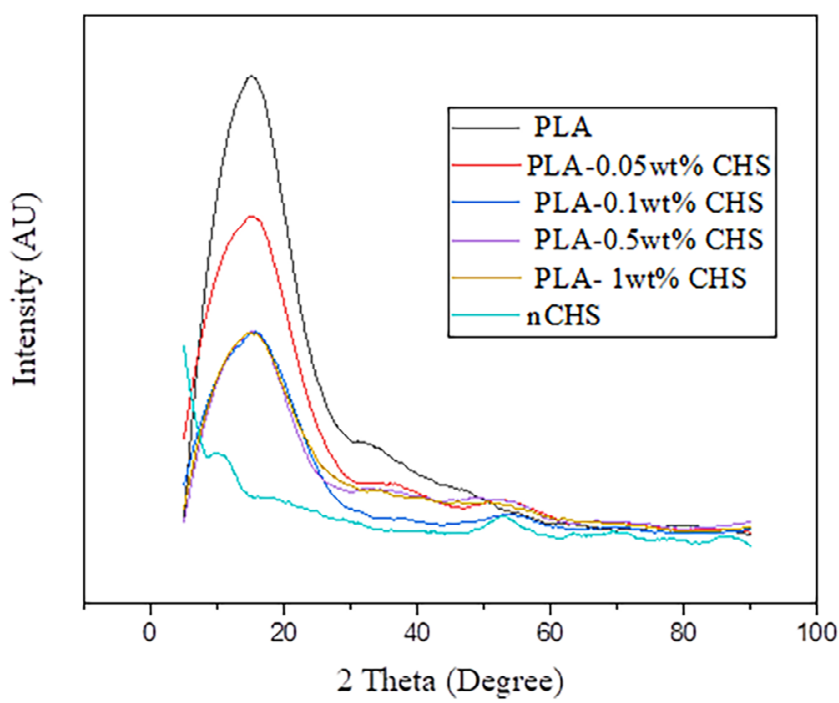

F I G U RE 7 The X-ray diffraction (XRD) patterns of the neat polylactic acid (PLA) membrane and nano chitosan (nCHS)/PLA nanocomposites [Color figure can be viewed at wileyonlinelibrary.com] strength seemed to be lowered. This is because of the aggregation of nCHS particles having large surface area and surface energy, which leads to the poor dispersion of nCHS in PLA matrix. ${ }^{[33]}$ In addition, these aggregates of nCHS can act as stress concentration centers which inhibit the stress transfer from the polymer matrix to the fillers. ${ }^{[33]}$ The good tensile properties at lower concentration of nCHS particles arises as a result of uniform stress distribution, minimized formation of stress-concentration centers, increased interfacial area for stress transfer from the polymer matrix to the fillers, decreased fiber diameter. ${ }^{[34,35]}$ These results indicated that nanocomposite fibers with lower nCHS content have less agglomeration, and finest fiber sizes would produce fibers with better mechanical properties. The SEM images provide a proof for this observation. Owing to the fact that the electrospun mats are non-woven fabrics, their mechanical properties are also influenced by several factors including composition, morphology of individual fibers and as well as interaction between

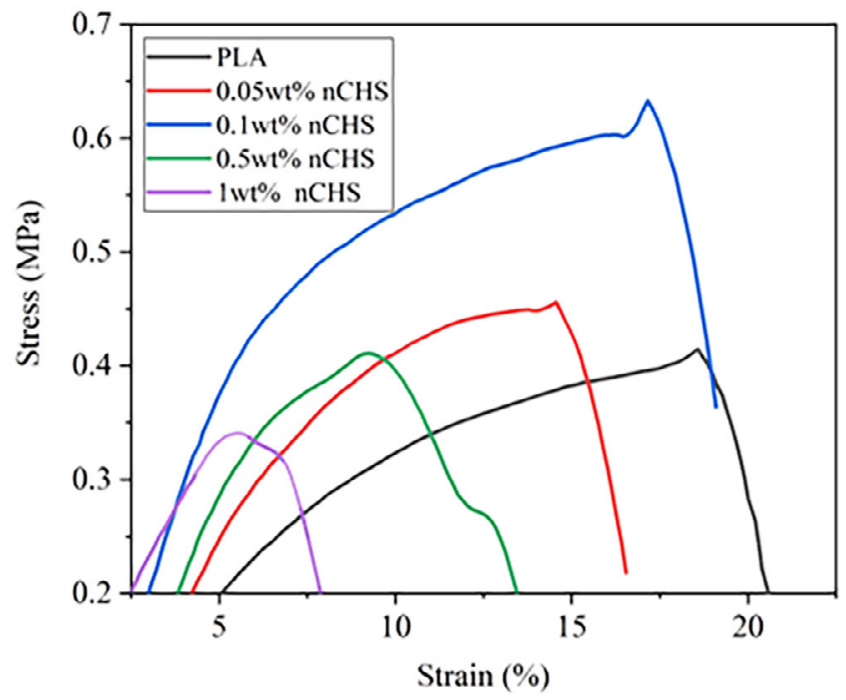

F I G U R E 8 Stress-strain curve of polylactic acid (PLA)-nano chitosan (nCHS) composites at different filler loading oriented distribution in the membrane [Color figure can be viewed at wileyonlinelibrary.com]

TABLE 1 Mechanical properties of PLA-nCHS composites

\begin{tabular}{llll}
$\begin{array}{l}\text { Fiber } \\
\text { content } \\
\text { (wt\%) }\end{array}$ & $\begin{array}{l}\text { Tensile } \\
\text { strength } \\
\text { (MPa) }\end{array}$ & $\begin{array}{l}\text { Young's } \\
\text { Modulus } \\
\text { (MPa) }\end{array}$ & $\begin{array}{l}\text { Elongation } \\
\text { at break } \\
(\%)\end{array}$ \\
\hline 0.00 & 0.40 & 4.05 & 20.5 \\
\hline 0.05 & 0.44 & 6.71 & 15.2 \\
\hline 0.10 & 0.60 & 15.04 & 18.0 \\
\hline 0.50 & 0.42 & 6.71 & 13.7 \\
\hline 1.00 & 0.33 & 7.09 & 8.13 \\
\hline
\end{tabular}

Abbreviations: nCHS, nano chitosan; PLA, polylactic acid. 


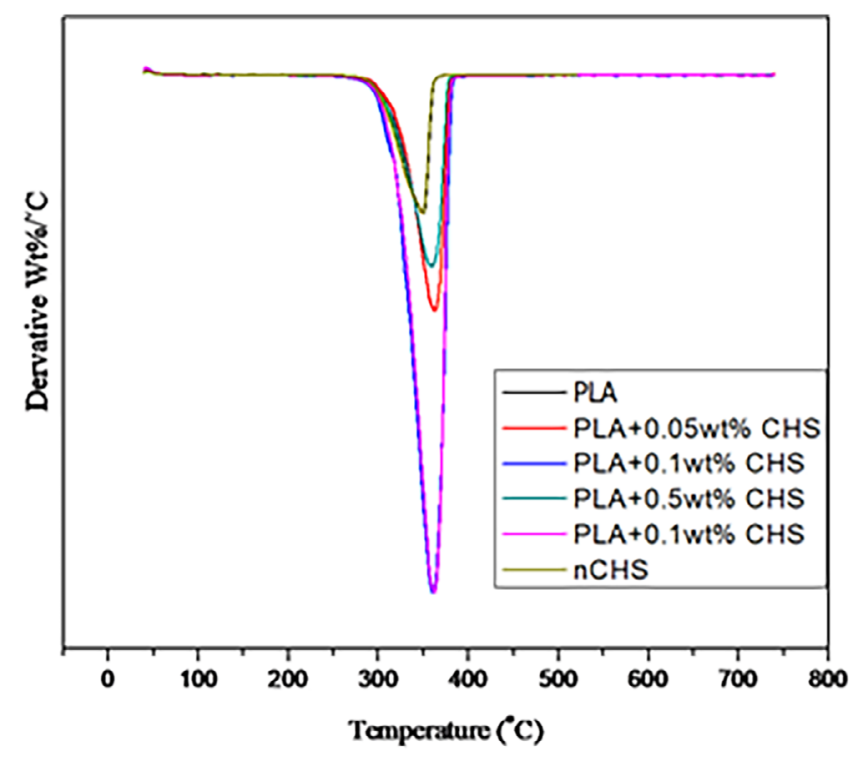

F I G U R E 9 Differential thermogravimetry (DTG) curve of polylactic acid (PLA),nano chitosan (nCHS), and PLA-nCHS composites [Color figure can be viewed at wileyonlinelibrary.com]

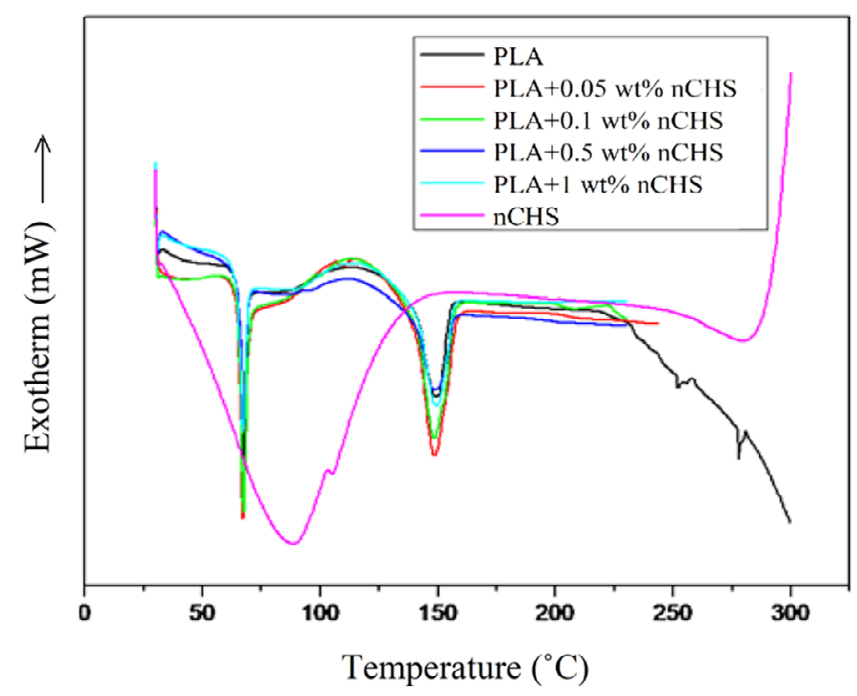

F I G U RE 10 Differential scanning calorimetry (DSC) thermogram of polylactic acid (PLA), nano chitosan (nCHS), and PLA-nCHS composites [Color figure can be viewed at wileyonlinelibrary.com]

fibers. In the case of PLA-nCHS fibers with 0.1 wt\% filler content showed the finest fiber sizes, and since many electrospun fibers form membranes, they can thus provide more contact and stronger cohesion among the fibers. ${ }^{[33]}$

\section{5 | Thermal properties}

Figure 9 represents the DTG curve for degradation of PLA, nCHS, and PLA-nCHS composites. The nCHS loses its moisture around $50-150^{\circ} \mathrm{C}$. The second thermal degradation due to the polymer decomposition was observed over a wide temperature range, from 250 to $340^{\circ} \mathrm{C}^{[22,36]}$ Only one degradation step was observed for the neat polymer and for the composites, and the thermal stability was better for the composites but did not change significantly with increasing fiber content. The degradation of nCHS in the composites was not observed as a separate step (Figure 9), and we assume that this was due to the low filler content and the good interaction between the nanoparticle and PLA. ${ }^{[37]}$

The glass transition, crystallization and melting of the samples are clearly observed in the DSC curves (Figure 10). The glass transition temperature of the PLA was around $60^{\circ} \mathrm{C}$ and was not changed by the addition of nCHS into it (PLA + nCHS composites). The nCHS content was too low and any anticipated influence that it may have had on the polymer chain mobility was dwarfed by the influence of the PLA crystals. A broad exothermic transition around $110^{\circ} \mathrm{C}$ (Figure 8) is due to the cold crystallization of PLA. ${ }^{[37]}$ Note that the intensity of the peak was decreased with the increase in the nCHS content. This indicates that the nanoparticles can either act as nucleation sites for the crystallization of the polymer or restrict the mobility of the polymer chains. ${ }^{[38]}$

\subsection{Zeta potential measurements}

Zeta potential is related to the quantity and dissociation of charged groups on the material surface and is a measure of stability of nanoparticles in the solution (Table 2). Presence of positively or negatively surface charged groups will result in a higher or lower zeta potential value, where higher zeta potential value (despite of the magnitude) refers to the stability of the suspension. ${ }^{[39]}$ Zeta potential value of PLA, CHS and composites are shown in Table 2. It was clear (from the value presented in the Table 2) that nCHS possess a high stability ( $\zeta=39.6$ ), which may be due to the ionization of free amino groups of chitosan in aqueous medium to ammonium ion $\left(-\mathrm{NH}_{2}\right.$ to $\left.-\mathrm{NH}_{3}^{+}\right)$. Note that the stability observed for PLA is very low. The composite showed a high zeta value compared to PLA because of the presence nCHS and is indicative of the better interaction of the nCHS and PLA.

\section{7 | Contact angle measurements}

Hydrophobicity as well as the surface energy characteristics of the composites was assessed by the contact angle measurements. Figure 11 represents the water 
T A B L E 2 Zeta potential results of nCHS, PLA, and PLA-nCHS

\begin{tabular}{|lc} 
Sample & Zeta potential value $(\mathbf{m V})$ \\
\hline nCHS & $39.6 \pm 4.7$ \\
\hline PLA & $3.36 \pm 0.64$ \\
\hline PLA-nCHS $(0.1 \mathrm{wt} \%)$ composite & $7 . \pm 2.8$ \\
\hline
\end{tabular}

Abbreviations: nCHS, nano chitosan; PLA, polylactic acid.

contact angles measured for electrospun membranes. Table 3 shows the contact angle values obtained for the composites with increased filler concentration. It was found that the contact angle values for 0.05 and $0.1 \mathrm{wt} \%$ nCHS composites showed more hydrophilic nature than the neat PLA. The results showed that when the nCHS content in the solutions increased from 0 to $0.1 \mathrm{wt} \%$, the water contact angles of the PLA nanofiber membranes decreased significantly from 77 to $73^{\circ}$. The increment of the nCHS content has decreased the fiber diameters and pore sizes of the modified membranes and hence increased the surface roughness, which can lead to a decrease of contact angle values, thereby increasing the wetting ability of the fibrous membrane. The surface free energy of PLA-nCHS composites were calculated and are shown in Table 3. According to the concept of wetting process, hydrophobic nature is observed when the solid-vapor interfacial energy is low and then tendency for spreading to eliminate the interface will be less. ${ }^{[40]}$ In order to reduce the surface energy of the system nanofillers should properly

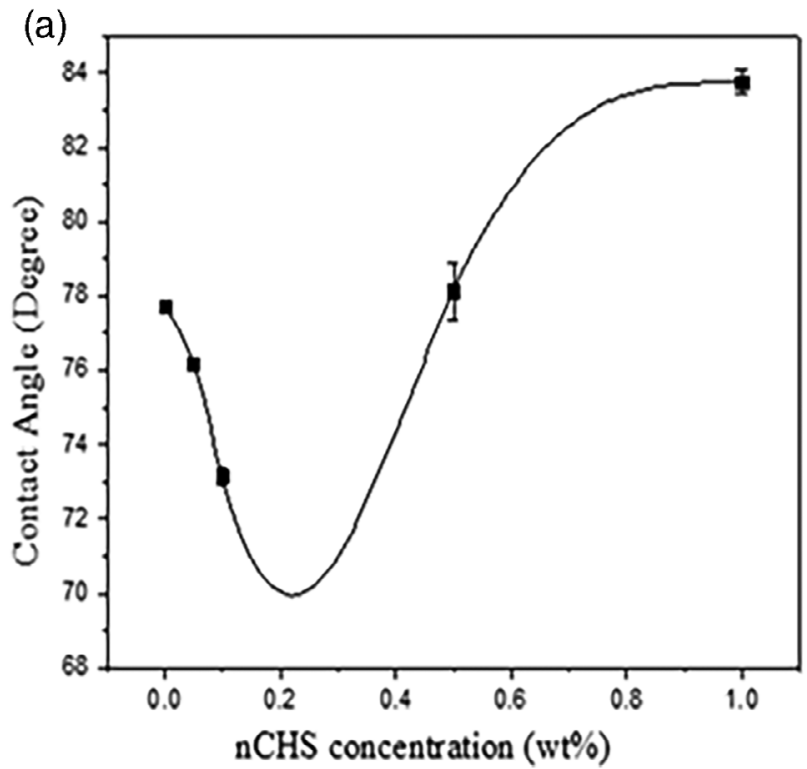

interact with polymer matrix. ${ }^{[41]}$ Composites of 0.05 and $0.1 \mathrm{wt} \% \mathrm{nCHS}$ shows significantly increased surface energy as compared to neat PLA, which might be due to the increase in free energy in mixing of two components at the interface.

\section{8 | Surface properties by inverse gas chromatography}

The electrospinning of the two biodegradable polymers lead to a makeover of the surface composition and morphology which strongly influence the surface properties, which can be analyzed by IGC. The neat PLA displayed a $\gamma_{s}^{d} \gamma_{s}^{d}$ value of $35.36 \mathrm{~mJ} / \mathrm{m}^{2}$ (Table 4) which agrees with the values reported in the literature. ${ }^{[24]}$ The PLA nanocomposites exhibit a lower $\gamma_{s}^{d} \gamma_{s}^{d}$ than the neat matrix, which decreased with the increased addition

T A B LE 3 The contact angle and surface free energy of PLA and PLA-nCHS composites

\begin{tabular}{|c|c|c|}
\hline Sample & $\begin{array}{l}\text { Contact angle } \\
\text { value (degree) }\end{array}$ & $\begin{array}{l}\text { Surface free } \\
\text { energy }\left(\mathrm{mJ} / \mathrm{m}^{2}\right)\end{array}$ \\
\hline PLA & $77.71^{\circ}$ & 33.05 \\
\hline PLA- $0.05 \mathrm{nCHS}$ & $76.14^{\circ}$ & 34.52 \\
\hline PLA-0.1 nCHS & $73.14^{\circ}$ & 37.39 \\
\hline PLA-0.5 nCHS & $78.12^{\circ}$ & 32.67 \\
\hline PLA-1 nCHS & $83.74^{\circ}$ & 27.64 \\
\hline
\end{tabular}

Abbreviations: nCHS, nano chitosan; PLA, polylactic acid.

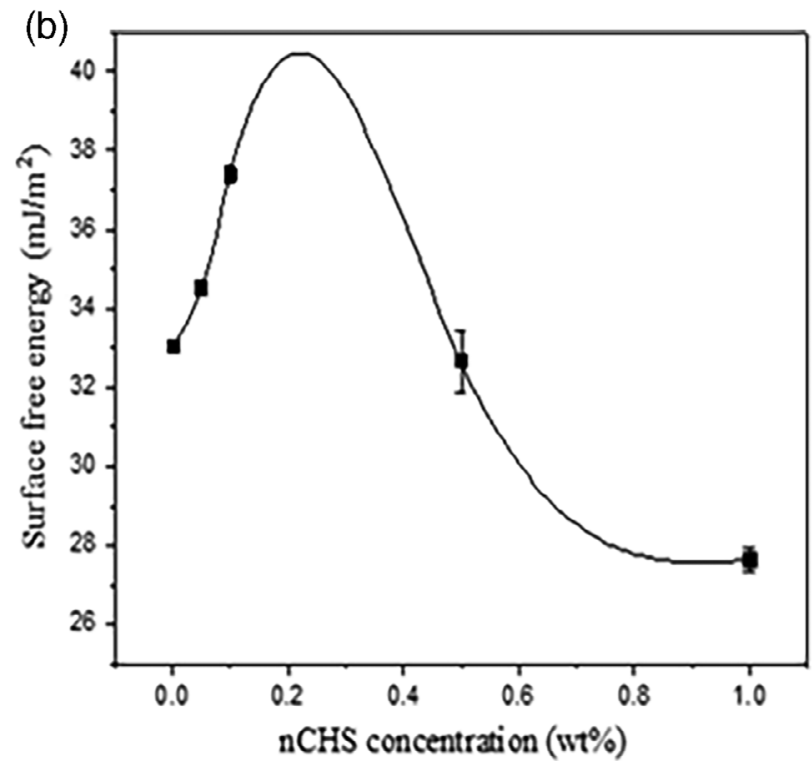

F I G U RE 11 (a) Contact angles measurements for polylactic acid (PLA), nano chitosan (nCHS), and PLA-nCHS; (b) Surface free energy measurements for PLA, nCHS, and PLA-nCHS 
T A B L E 4 IGC results of neat PLA and PLA with 0.1 and $1 \mathrm{wt} \% \mathrm{nCHS}$, at $25^{\circ} \mathrm{C}$

\begin{tabular}{lccc} 
& PLA & PLA/nCHS 0.1 wt\% & PLA/nCHS 1 wt\% \\
\hline$\gamma_{s}^{\boldsymbol{d}}\left(\mathrm{mJ} / \mathrm{m}^{2}\right)$ & 35.36 & 34.53 & 32.86 \\
$\boldsymbol{K}_{\boldsymbol{a}}$ & 0.08 & 0.08 & 0.13 \\
$\boldsymbol{K}_{\boldsymbol{b}}$ & 0.15 & 0.19 & 0.27 \\
$\boldsymbol{K}_{\boldsymbol{b}} / \boldsymbol{K}_{\boldsymbol{a}}$ & 1.88 & 2.38 & 2.08 \\
\hline
\end{tabular}

Abbreviations: IGC, inverse gas chromatography; nCHS, nano chitosan; PLA, polylactic acid.

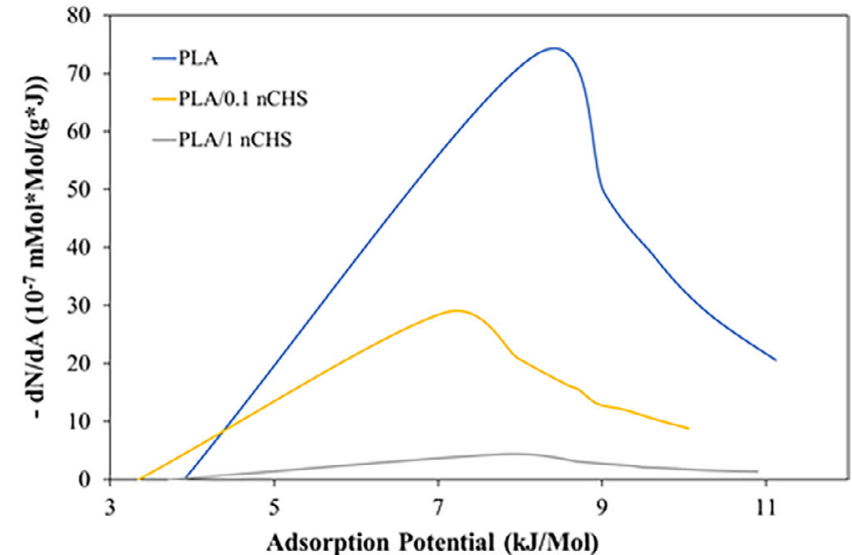

F I G U R E $12 n$-Octane heterogeneity profile to neat polylactic acid (PLA) and PLA composites, at $25^{\circ} \mathrm{C}$ [Color figure can be viewed at wileyonlinelibrary.com]

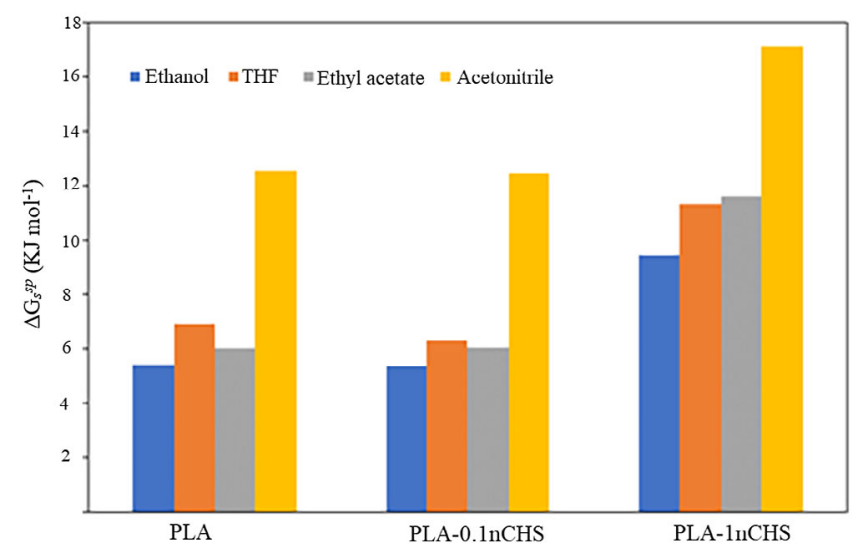

F I G URE 13 Specific free energy of adsorption $\left(\Delta G_{d}^{s p}\right)$ to neat polylactic acid (PLA) and PLA composites, at $25^{\circ} \mathrm{C}$ [Color figure can be viewed at wileyonlinelibrary.com]

nCHS into the PLA. This decrease can be associated with the decrease in the number and/or in the energy of the active sites determined through the Brunauer-EmmetTeller (BET) isotherms measurements with $n$-octane. The energetic profile of nanocomposites showed in Figure 12 confirms this: a decrease of $\gamma_{s}^{d} \gamma_{s}^{d}$ value is justified by the decrease in the number of active sites and in the active

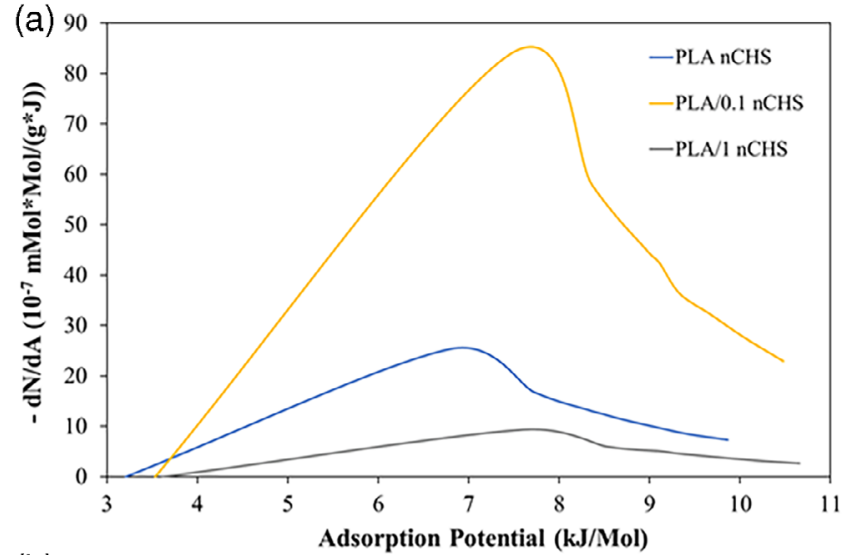

(b)

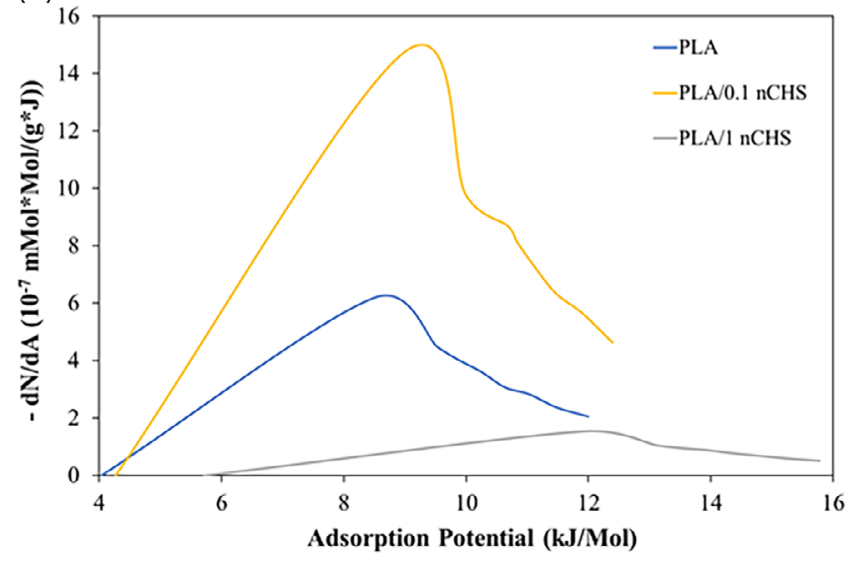

F I G U RE 14 Heterogeneity profile with (a) tetrahydrofuran and (b) to neat polylactic acid (PLA) and PLA composites, at $25^{\circ} \mathrm{C}$ [Color figure can be viewed at wileyonlinelibrary.com]

site's energy. Based on these results, the PLA nanocomposites should become more hydrophilic: a lower $\gamma_{s}^{d} \gamma_{s}^{d}$ is an indication that the $n$-alkane probe molecules had lower interaction with the non-polar groups on the surface of PLA nanocomposites. These results are in agreement with the data obtained from the contact angle measurements.

Four polar probe molecules (tetrahydrofuran, ethanol, ethyl acetate, and acetonitrile) were injected to determine the specific component of the surface energy, $\Delta G_{d}^{s p}$, of all nanocomposites at $25^{\circ} \mathrm{C}$ (Figure 13). Neat PLA and PLA/nCHS nanocomposites exhibited strong 


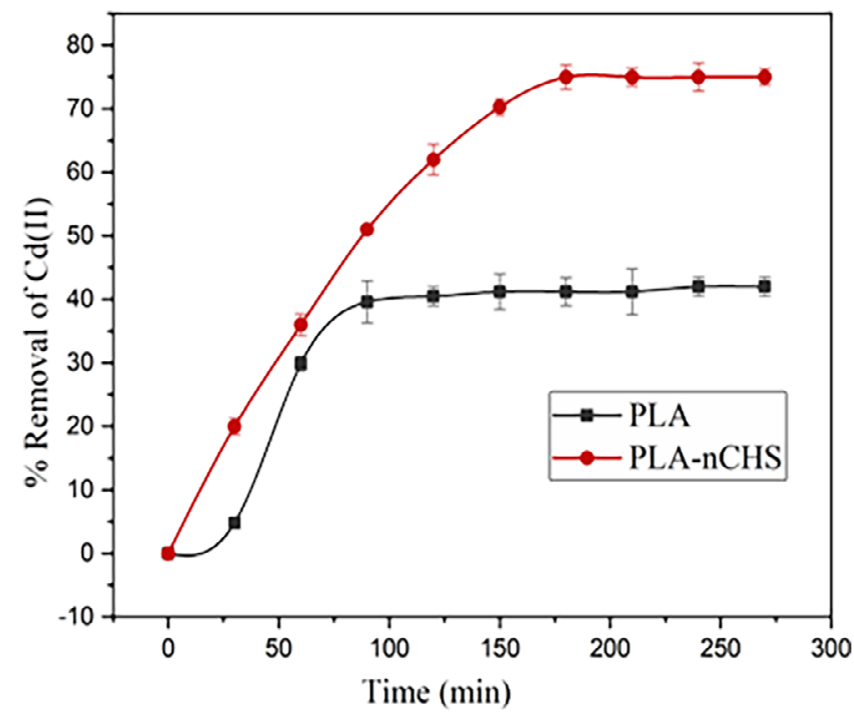

F I G U RE 15 Percentage of cadmium(II) removal by polylactic acid (PLA) and PLA-nano chitosan (nCHS) composite as a function of contact time [Color figure can be viewed at wileyonlinelibrary.com]

interactions with acetonitrile, an amphoteric probe molecule, which indicated the presence of both acid and base groups in the materials surface. High content of chitosan nanoparticles into PLA network leads to an increase in the $\Delta G_{s}^{s p}$ value which indicates the increase of the polar groups on the surface of the nanocomposites. These results are corroborated with the obtained energetic profile from the acid probe molecule (ethanol) and basic probe molecule (tetrahydrofuran) showed in Figure 14. In both energetic profiles an increase in the number of active sites at higher energy was observed with the nCHS incorporation in the PLA. The Lewis acid-base constants, $K_{A}$ and $K_{B}$, were determinate through the $\Delta G_{s}^{s p}$ value and are presented in Table 4. Compared with the neat PLA, all the nanocomposites exhibited an increase in $K_{A}$ and $K_{B}$ constant, which indicates that the surface became more basic with the incorporation of nCHS which is more evident at $0.1 \mathrm{wt} \% \mathrm{nCHS}\left(K_{B} / K_{A}=2.38\right)$. Given the lack of hydroxyl groups on PLA network to form hydrogen bonds with the chitosan amino groups, the basic character of the composites has intensified with the increase in nCHS addition.

\subsection{Atomic absorption spectroscopy (AAS) analysis}

AAS analysis was done to compare the heavy metal $\left(\mathrm{Cd}^{2+}\right)$ removal efficiency of the composite with neat PLA. The percentage of cadmium(II) removal by PLA and PLAnCHS composite as a function of contact time is depicted in Figure 15. The sorption of cadmium by the sorbent was found to be very fast in the initial stage and showed a gradual decrease thereafter and reached an equilibrium value in approximately approximately 120 minutes. This indicates the saturation of abundant vacant binding sites, and after saturation point all these sites were in an occupied state. The PLA fiber mats also showed an adsorption capacity for the $\mathrm{Cd}^{2+}$ ion due to the secondary porous structures of the PLA membrane. The adsorption of metal ions on the bio sorbent surface depends on the availability of metal ion around the microenvironment of the bio sorbent and the electrostatic interaction between them at the sorption sites. ${ }^{[42]}$ Nano chitosan can act as a bio sorbent and the porous nature of electrospun membrane will facilitate the adsorption capacity of the membrane. The hybrid PLA/nCHS fibers possess high surface roughness due to the nanometer-scale pores distributed on the surface of the fibers, and small fiber diameter, which would provide the relevant fibrous membrane with a higher probability of capturing the target particles, finally enhancing the filtration performance.

\section{4 | CONCLUSION}

nCHS act as a reinforcement and increase the sorption capacity of electrospun PLA membrane. In addition to this the addition of nCHS decreased the diameter of the fibers and also decreased the pore size of the secondary porous structure upon $0.1 \mathrm{wt} \%$ addition of nCHS. The thermal and FTIR studies did not give any indication for the incorporation of nCHS into the PLA membranes, which may be due to the low chitosan content. The tensile strength of the composite was improved upto $0.1 \mathrm{wt} \%$ of nCHS is an indication of uniform distribution of nanoparticles and nCHS decreased the crystallinity of the composite membranes. Zeta potential analysis indicated the high stability of the composites. Contact angle measurements suggested that the PLA-nCHS system become hydrophilic and the surface energy of the system increased upto $0.1 \mathrm{wt} \%$ due to the increase in free energy in mixing of two components at the interface. IGC analysis revealed that nCHS incorporation into the PLA makes the surface more hydrophilic and with a stronger basic character. The porous natures of electrospun membranes facilitate its use as a filter for heavy metals from water. The results suggested that the composite offer about $70 \% \mathrm{Cd}^{2+}$ removal. Hence, we can use PLA-nCHS composite as an effective material for the removal of $\mathrm{Cd}^{2+}$ from wastewater.

\section{ORCID}

Laly A. Pothen (1) https://orcid.org/0000-0001-9723-0854 


\section{REFERENCES}

[1] S. Daer, J. Kharraz, A. Giwa, S. W. Hasan, DES 2015, 367, 37. https://doi.org/10.1016/j.desal.2015.03.030.

[2] Hu, N., Cheney, M. A., Hanifehpour, Y., Joo, S. W., \& Min, B. (2017). Synthesis, characterization, and catalytic performance of $\mathrm{Sb} 2$ se 3 nanorods.

[3] A. P. Nikalje, Med. Chem. 2015, 5(2), 81. https://doi.org/10. 4172/2161-0444.1000247.

[4] S. Sikarwar, B. C. Yadav, Sensors Actuators A Phys. 2015, 233, 54. https://doi.org/10.1016/j.sna.2015.05.007.

[5] R. Gopal, S. Kaur, Z. Ma, C. Chan, S. Ramakrishna, T. Matsuura, J. Membr. Sci. 2006, 281(1-2), 581. https://doi. org/10.1016/j.memsci.2006.04.026.

[6] L. Huang, S. S. Manickam, J. R. McCutcheon, J. Membr. Sci. 2013, 436, 213.

[7] C. Cui, C. Xiang, L. Geng, X. Lai, R. Guo, Y. Zhang, H. Xiao, J. Lan, S. Lin, S. Jiang, J. Alloys Compd. 2019, 788, 1246.

[8] A. Haider, S. Haider, I.-K. Kang, Arab. J. Chem. 2018, 11(8), 1165.

[9] Y. Zhan, X. Guan, E. Ren, S. Lin, J. Lan, J. Polym. Res. 2019, 26(6), 145.

[10] C. T. Lim, Prog. Polym. Sci. 2017, 70, 1.

[11] R. Passos de Oliveira Santos, P. Fernanda Rossi, L. A. Ramos, E. Frollini, Polymers 2018, 10(5), 538.

[12] J. An, K. Wang, S. Chen, M. Kong, Y. Teng, L. Wang, C. Song, D. Kong, S. Wang, J. Bioact. Compat. Polym. 2015, 30(2), 209.

[13] B. Azimi, P. Nourpanah, M. Rabiee, S. Arbab, J. Eng. Fabr. Fibers 2014, 9(3), 74.

[14] A. M. Ziemba, K. P. Lane, I. M. San Segundo, A. R. D'Amato, A. K. Mason, R. J. Sexton, et al., ACS Biomater. Sci. Eng. 2018, 4 (5), 1491.

[15] V. H. Sangeetha, H. Deka, T. O. Varghese, S. K. Nayak, Polym. Compos. 2018, 39(1), 81.

[16] O. Valerio, M. Misra, A. K. Mohanty, RSC Adv. 2017, 7(61), 38594.

[17] F. E. Ahmed, B. S. Lalia, R. Hashaikeh, Desalination 2015, 356, 15.

[18] S. T. Lin, L. Kimble, D. Bhattacharyya, Polymer blends and composites for biomedical applications. in Biomaterials for Implants and Scaffolds, Springer, Berlin, Germany 2017, p. 195.

[19] A. Anitha, S. Sowmya, P. T. S. Kumar, S. Deepthi, K. P. Chennazhi, H. Ehrlich, M. Tsurkan, R. Jayakumar, Prog. Polym. Sci. 2014, 39(9), 1644.

[20] W. S. Wan Ngah, L. C. Teong, M. A. K. M. Hanafiah, Carbohydr. Polym. 2011, 83(4), 1446. https://doi.org/10.1016/j. carbpol.2010.11.004.

[21] P. Calvo, C. Remuñan-López, J. L. Vila-Jato, M. J. Alonso, Pharm. Res. 1997, 14(10), 1431.

[22] M. S. Sivakami, T. Gomathi, J. Venkatesan, H. S. Jeong, S. K. Kim, P. N. Sudha, Int. J. Biol. Macromol. 2013, 57(March 2013), 204. https://doi.org/10.1016/j.ijbiomac.2013.03.005.

[23] M. K. M. Haafiz, A. Hassan, Z. Zakaria, I. M. Inuwa, M. S. Islam, M. Jawaid, Carbohydr. Polym. 2013, 98(1), 139.
[24] M. S. Thomas, P. K. S. Pillai, M. Faria, N. Cordeiro, H. Barud, S. Thomas, L. A. Pothen, J. Mater. Sci. Mater. Med. 2018, 29(9), 137. https://doi.org/10.1007/s10856-018-6146-1.

[25] R. M. Nezarati, M. B. Eifert, E. Cosgriff-Hernandez, Tissue Engineering Part C: Methods 2013, 19(10), 810. https://doi.org/ 10.1089/ten.tec.2012.0671.

[26] P. Neogi, AICHE J. 1983, 29(3), 402.

[27] P. Van de Witte, P. J. Dijkstra, J. W. A. Van den Berg, J. Feijen, J. Membr. Sci. 1996, 117(1-2), 1.

[28] C. L. Casper, J. S. Stephens, N. G. Tassi, D. B. Chase, J. F. Rabolt, Macromolecules 2004, 37(2), 573.

[29] R. Inai, M. Kotaki, S. Ramakrishna, Nanotechnology 2005, 16 (2), 208. https://doi.org/10.1088/0957-4484/16/2/005.

[30] W. Liu, M. Graham, E. A. Evans, D. H. Reneker, J. Mater. Res. 2002, 17(12), 3206.

[31] X. Zong, K. Kim, D. Fang, S. Ran, B. S. Hsiao, B. Chu, Polymer 2002, 43(16), 4403. https://doi.org/10.1016/S0032-3861(02) 00275-6.

[32] L. Qi, Z. Xu, Colloids Surf. A Physicochem. Eng. Asp. 2004, 251 (1-3), 183. https://doi.org/10.1016/j.colsurfa.2004.10.010.

[33] R. Augustine, E. A. Dominic, I. Reju, B. Kaimal, N. Kalarikkal, S. Thomas, RSC Adv. 2014, 4(47), 24777. https://doi.org/10. 1039/c4ra02450h.

[34] C. T. Lim, E. P. S. Tan, S. Y. Ng, Appl. Phys. Lett. 2008, 92(14), 141908.

[35] M. Moniruzzaman, J. Chattopadhyay, W. E. Billups, K. I. Winey, Nano Lett. 2007, 7(5), 1178.

[36] E. Szymańska, K. Winnicka, Mar. Drugs 2015, 13(4), 1819.

[37] J. P. Mofokeng, A. S. Luyt, T. Tábi, J. Kovács, J. Thermoplast. Compos. Mater. 2012, 25(8), 927.

[38] J. D. Menczel, L. Judovits, R. B. Prime, H. E. Bair, M. Reading, S. Swier, Differential scanning calorimetry (DSC). in Thermal Analysis of Polymers: Fundamentals and Applications, John Wiley \&Sons, Hoboken, New Jersey 2009, p. 7.

[39] I. Ostolska, M. Wiśniewska, Colloid Polym. Sci. 2014, 292(10), 2453.

[40] G. Kumar, K. N. Prabhu, Adv. Colloid Interf. Sci. 2007, 133(2), 61.

[41] J. P. Jose, J. Abraham, H. J. Maria, K. T. Varughese, S. Thomas, Macromol. Symp. 2016, 366(1), 66.

[42] S. S. Pillai, B. Deepa, E. Abraham, N. Girija, P. Geetha, L. Jacob, M. Koshy, Ecotoxicol. Environ. Saf. 2013, 98(Ii), 352. https://doi.org/10.1016/j.ecoenv.2013.09.003.

How to cite this article: Thomas MS, Pillai PKS, Faria M, et al. Polylactic acid/nano chitosan composite fibers and their morphological, physical characterization for the removal of cadmium(II) from water. J Appl Polym Sci. 2020;e48993. https:// doi.org/10.1002/app.48993 